\title{
Study of RANS Turbulence Models for Turbulent Wake Flow in Adverse Pressure Gradient
}

\author{
Tobias Knopp $^{1(\bowtie)}$, Paul Korsmeier ${ }^{1}$, Mikael Strelets $^{2}$, and Ekaterina Guseva ${ }^{2}$
}

1 DLR, Institut für Aerodynamik und Strömungstechnik, Bunsenstr. 10, 37073 Göttingen, Germany

\{tobias.knopp, paul.korsmeier\}@dlr.de

2 Saint Petersburg Polytechnic University, Saint Petersburg, Russia

\begin{abstract}
We present an analysis of RANS turbulence models for a new turbulent wake flow case in adverse pressure gradient. The reference data are obtained from an LES. RANS simulations using the Spalart-Allmaras model, the SST model and the SSG/LRR- $\omega$ model show a tendency to underpredict the onset of flow reversal in the wake. The comparison with the LES data reveals that the SSG/LRR- $\omega$ model underpredicts the turbulent transport of the Reynolds stresses and overpredicts the ratio of production to dissipation. Therefore two modifications of the SSG/LRR- $\omega$ model are studied, i.e., the modification of the coefficient of the gradient diffusion hypothesis for the turbulent transport of the Reynolds stresses and the sensitization of the dissipation rate to irrotational strains. Both modifications improve the agreement with the reference data.
\end{abstract}

\section{Introduction}

The aerodynamic performance of a multi-element high-lift wing depends not only on the separation behaviour of the boundary layers, but also on the behaviour of the turbulent wake flows generated by the slat and the main wing element when subjected to a strong adverse pressure gradient, see [13]. The wake flow can be prone to flow reversal, which can lead to wing stall even if the boundary layer flow remains attached. Several investigations in the past showed that standard RANS models are not able to predict this flow phenomenon accurately. The SST model was found to underpredict the tendency for flow reversal for the test-case by Driver \& Mateer, see [3], and this was attributed to the tendency to overpredict the Reynolds stresses. Similar observations were found in [2] for the SST model and for the SSG/LRR- $\omega$ model for the test-case by Driver \& Mateer, and by Tummers et al. for their own experiment [12].

Albeit both experiments by Driver \& Mateer and by Tummers et al. provide detailed data, the reasons for the deviation between the RANS simulations and the experiments cannot be fully explained, for which different reasons are possible. The first reason is that the boundary conditions in the experiments are

(C) The Author(s), under exclusive license to Springer Nature Switzerland AG 2021 A. Dillmann et al. (Eds.): STAB/DGLR Symposium 2020, NNFM 151, pp. 58-67, 2021. https://doi.org/10.1007/978-3-030-79561-0_6 
not defined exactly. In both cases flow control was applied on the wind tunnel walls, which generate the adverse pressure gradient, but the information given by the authors is not sufficient to reproduce the boundary conditions in RANS simulations. The second issue is that using experimental techniques alone, it is not possible to measure all terms of the turbulent kinetic energy equation and of the Reynolds stress equation. The third issue is that the experiments are likely to be influenced by side-wall effects, especially when flow reversal takes place. The displacement effect of the side-walls causes a mean flow acceleration in the centerplane, and this cannot be accounted for in a 2D simulation. On the other hand, in 3D simulations of the wind-tunnel experiments of wake-flow in adverse pressure gradient cited above (despite the lack of detailed information about the flow actuation), the onset of flow separation of the side-wall boundary layers in adverse pressure gradient plays a significant role and can hardly be distinguished from the onset of flow reversal in the centerline of the wake.

For these reasons, a joint German-Russian research project "Wake Flows in Adverse Pressure Gradient", supported by DFG and RBRF, was started in 2017. A new test-case was designed with exactly specified boundary conditions. The test-case was studied experimentally [1] and numerically (using Large-Eddy Simulation (LES)) [6]. The comparison between experiment and LES will be described in a separate paper. The LES provides all terms of the Reynolds stress equation and of the turbulent kinetic energy equation. In particular, unique data for the turbulent transport term and for the dissipation $\epsilon$ were obtained. Moreover the numerical experiment provides a well-defined test case with precisely known boundary conditions. The flow of the numerical experiment is statistically two-dimensional and free of side-wall effects.

\section{Test Case Description}

The test-case was designed to achieve a strong adverse pressure gradient in the wake with a significant reduction of the centerline velocity and a small region of flow-reversal. At the same time, the test-case should be suitable for RANS simulations, i.e., the boundary conditions should be well-defined and without the use of active flow control, e.g., synthetic jets, to avoid flow separation on the geometry parts which generate the adverse pressure gradient. Moreover, the aim was to achieve a macroscopically statistically steady flow, which was presumably not fully met, for example, for the experiment by Driver and Mateer, see [6].

Regarding the geometry of the test case in Fig. 1, a flat plate (FP) of length $L=1.058 \mathrm{~m}$ and thickness of $0.02 \mathrm{~m}$ with a rounded leading edge and a linearly tapered trailing edge was used as a wake generator. On both sides of the symmetry plane, two thin liner foils (LF1 and LF2) act as a diffusor-stabilizer arrangement to impose the APG and avoid unsteady vortex shedding. The slot between the first and second liner foil serves as a passive mechanism to prevent flow separation on the liner foil. Active boundary layer control techniques were not necessary. This yields well-defined and stationary boundary conditions for the simulations. The origin of the coordinate system is located at the trailing 
edge of the splitter plate. The vertical distance $h$ of the liner foil leading edge to the symmetry plane was varied to change the strength of the pressure gradient. The present publication shows the results for $h=0.07 \mathrm{~m}$ (moderately strong APG, wake at the verge of flow reversal) and for $h=0.05 \mathrm{~m}$ (strong APG, flow reversal in the wake) for $R e_{L}=3.2 \times 10^{6}$ based on the onflow velocity $U_{\text {ref }}$ and the length of the splitter plate $L$.

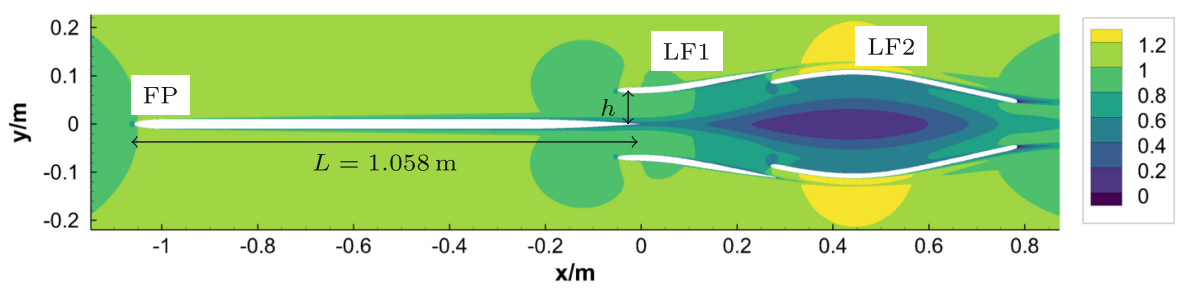

Fig. 1. Geometric setup and ratio of local to onflow velocity $U / U_{\text {ref }}$ for $h=0.07 \mathrm{~m}$

The reference data were obtained with the use of a scale-resolving simulation of the considered flow. Specifically, a zonal approach to turbulence representation was employed (see Fig. 2). It includes SST RANS computation of the initial part of the flat plate boundary layer and Improved Delayed Detached-Eddy Simulation (IDDES) [11] of the rest part of the flow. In the downstream part of the plate the IDDES functions as an LES with wall modelling (WMLES) while in the wake it performs as a pure LES. For triggering the WMLES branch of IDDES, the Volume Synthetic Turbulence Generator (VSTG) [10] is used, in order to create artificial turbulence at the inlet of the IDDES sub-domain needed for rapid transition from fully-modeled turbulence in the RANS subdomain to mostly-resolved turbulence in the IDDES sub-domain, see Fig. 3. The simulation was performed with the use of the in-house high-order finite-volume structured multi-block code NTS [9]. The grid used in the simulation has about 50 million cells and satisfies commonly accepted requirements for RANS-LES computations. Its sufficiency is supported by the grid-sensitivity study.

For the specifics of the wind-tunnel experiment we refer to [1].

\section{Numerical Setup for RANS Simulations}

The present simulations were performed using the DLR TAU code, a compressible finite volume solver for unstructured and hybrid meshes, using a secondorder accurate central scheme for the spatial discretisation and a local time stepping. Low Mach number preconditioning of the Rossow-Swanson type was employed.

At the inflow boundary, the reservoir pressure inflow condition was used. The reference values in the TAU code were set to $R e_{L}=3.2 \times 10^{6}$ based on the onflow (reference) velocity $U_{\text {ref }}=50 \mathrm{~m} / \mathrm{s}$, and the reference temperature was 


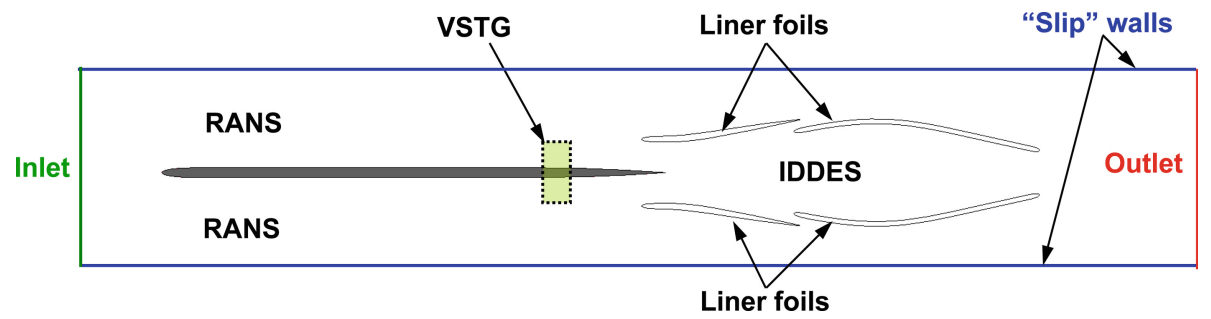

Fig. 2. Schematic of the zonal RANS-IDDES computational setup.

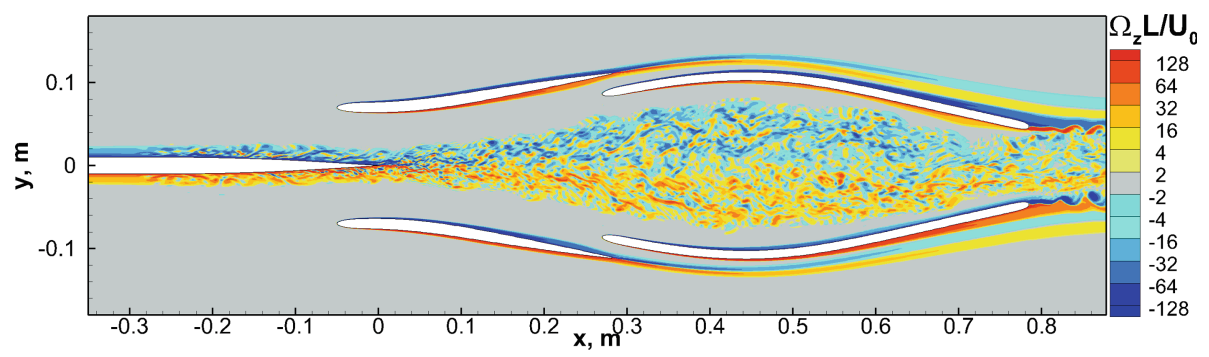

Fig. 3. Snapshot of the out-of-plane component $\Omega_{z}$ of the vorticity vector.

$T_{\text {ref }}=310.15 \mathrm{~K}$. The exit-pressure outflow condition was used at the outflow boundary, and the Euler wall condition at the upper and lower wind tunnel walls. The viscous surfaces of the flat plate and of all airfoils were fully turbulent.

The RANS meshes were generated using the Pointwise mesh generator. The spacing in streamwise direction is $\Delta x / L=0.006$ on the flat plate and $\Delta x / L \leq$ 0.002 in the wake. The wall-distance of the first grid point above the viscous walls $y^{+}$are below one on the flat plate and on the liner foils.

\section{RANS Turbulence Modelling}

For the RANS simulations, both eddy-viscosity models and differential Reynolds stress models were used. Regarding the turbulent viscosity models, we used the Spalart-Allmaras (SA) and the Menter Shear-Stress Transport model (SST) both without and with the extensions for rotation and curvature (RC) and the quadratic constitutive relation (QCR). For the differential Reynolds stress models we used the SSG/LRR- $\omega$ model. The transport equation for the Reynolds stress tensor reads as

$$
\frac{\partial\left(\bar{\varrho} R_{i j}\right)}{\partial t}+\frac{\partial\left(\bar{\varrho} R_{i j} U_{k}\right)}{\partial x_{k}}=\bar{\varrho}\left(P_{i j}+\Pi_{i j}-\epsilon_{i j}+D_{i j}\right) .
$$

The arising terms and their modelling are defined in Table 1 for the SSG/LRR- $\omega$ model. A single blending function (borrowed from the Menter SST model) is used 
Table 1. The terms of the SSG/LRR- $\omega$ model and their modelling.

\begin{tabular}{l|l|l}
\hline Name & Term & Model \\
\hline Production & $\bar{\varrho} P_{i j}$ & Unmodeled \\
Dissipation & $\bar{\varrho} \epsilon_{i j}$ & Menter baseline for $\bar{\varrho} \omega ; \epsilon=C_{\mu} k \omega ; \bar{\varrho} \epsilon_{i j}=2 \bar{\varrho} \epsilon \delta_{i j} / 3$ \\
Pressure-strain & $\bar{\varrho} \Pi_{i j}$ & $\begin{array}{l}\text { SSG in outer part of boundary layers, LRR in inner wall } \\
\text { layer } \\
\text { Diffusion }\end{array}$ \\
\hline
\end{tabular}

for all coefficients. For the turbulent transport (part of $\bar{\varrho} D_{i j}$ ), the generalised gradient diffusion hypothesis (GGDH) is used:

$$
\bar{\varrho} D_{i j}=\frac{\partial}{\partial x_{m}}\left[\left(\bar{\mu} \delta_{m n}+C_{\mathrm{GGDH}} \frac{\bar{\varrho} k R_{m n}}{\epsilon}\right) \frac{\partial R_{i j}}{\partial x_{n}}\right]
$$

For more details, see [4] and references therein.

\subsection{Sensitization to Pressure Gradients}

In [7], the sensitization of the dissipation equation to irrotational strains by means of an additional term in the $\epsilon$ equation was proposed. The present work uses the corresponding term

$$
S_{\omega, 4}=-C_{\epsilon, 4}^{*} \frac{\omega}{k}\left(\overline{u_{s} u_{s}} \frac{\partial U_{s}}{\partial x_{s}}+\overline{u_{n, 1} u_{n, 1}} \frac{\partial U_{n, 1}}{\partial x_{n, 1}}+\overline{u_{n, 2} u_{n, 2}} \frac{\partial U_{n, 2}}{\partial x_{n, 2}}\right)
$$

which is based on the works $[5,8]$. Here, $C_{\epsilon, 4}^{*}=1.16$ and $x_{s}$ is the streamline direction that forms an orthonormal frame with the coordinate directions $x_{n, 1}$ and $x_{n, 2}$.

\subsection{Calibration of the GGDH Using the LES Data}

The LES data provide all terms of the TKE balance, in particular the exact data for the turbulent transport term $D_{k}^{t}$ from the triple correlations. We recall that the gradient diffusion hypothesis $(\mathrm{GDH})$ for the $k$-equation considers the diffusive transport term $D_{k}=D_{k}^{t}+D_{k}^{p}+D_{k}^{\nu}$ with the turbulent part $D_{k}^{t}$, the fluctuating pressure part $D_{k}^{p}$, and the viscous part $D_{k}^{\nu}$ given by

$$
D_{k}^{t}=-\frac{\partial}{\partial x_{i}} \frac{\overline{u_{i}^{\prime} u_{j}^{\prime} u_{j}^{\prime}}}{2}, \quad D_{k}^{p}=-\frac{\partial}{\partial x_{i}} \frac{\overline{u_{i}^{\prime} p^{\prime}}}{\varrho}, \quad D_{k}^{\nu}:=\nu \frac{\partial}{\partial x_{i}} \frac{\partial k}{\partial x_{i}}
$$

and models the sum of the three terms as

$$
D_{k}=\frac{\partial}{\partial x_{i}}\left(\left(\nu+C_{k} \nu_{t}\right) \frac{\partial k}{\partial x_{i}}\right)
$$


In the standard models like the SST model, $C_{k}=1$ and $\nu_{t}$ is the eddy viscosity. In this work the calibration for $C_{k}$ is revisited for wake flows at APG. From the LES data we evaluate $D_{k}$ from (5). Here the eddy viscosity is computed from the relation

$$
\nu_{t}=\frac{-\overline{u^{\prime} v^{\prime}}}{\frac{\partial U}{\partial y}+\frac{\partial V}{\partial x}+10^{-22}},
$$

The comparison at $x=0.2 \mathrm{~m}$ yielded that $C_{k}=2$ is more suitable than $C_{k}=1$ which is shown in Fig. 4. The analogous modification of the generalized gradient diffusion hypothesis coefficent $C_{\mathrm{GGDH}}$ (cf. (2)) in the SSG/LRR- $\omega$ model was thus implemented and activated for $x \geq 0.1 \mathrm{~m}$. Those results are denoted throughout the rest of this paper as "GGDH mod fac 2.0".
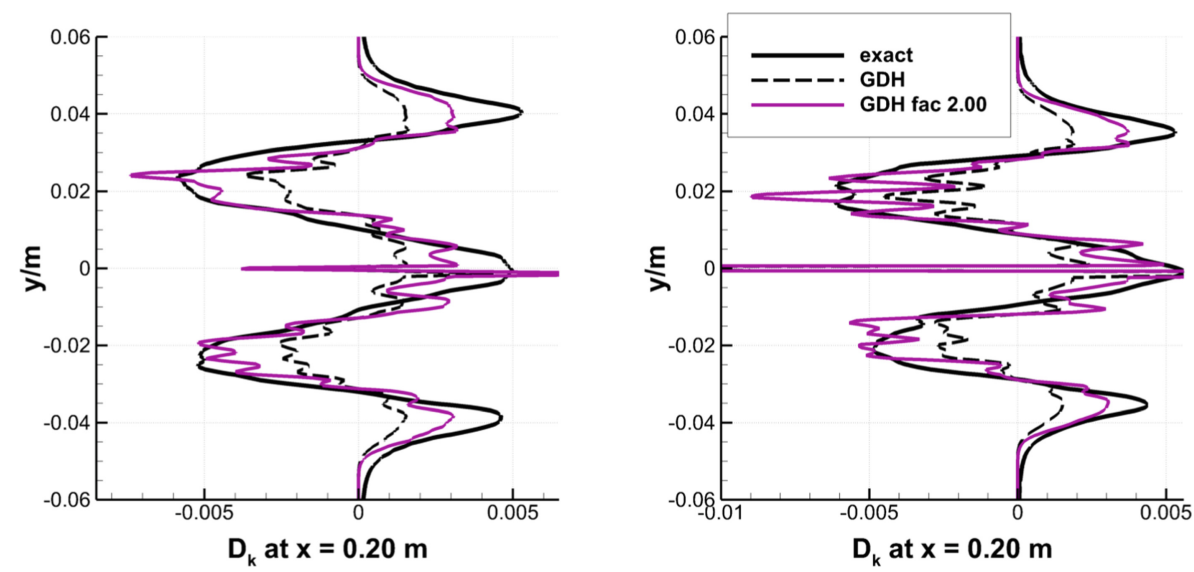

Fig. 4. Comparison of 'exact' TKE transport term $D_{k}\left(\right.$ in $\left.\left[\mathrm{m}^{2} \mathrm{~s}^{-3}\right]\right)$ from the LES data and its modelings according to (5) with $\nu_{t}$ defined in (6) and either $C_{k}=1$ (standard $\mathrm{GDH}$ ) or $C_{k}=2$ (modified GDH); for $h=5 \mathrm{~cm}$ (left) and $h=7 \mathrm{~cm}$ (right)

\section{Results}

\subsection{Mean Velocity}

First the mean velocity $U_{\mathrm{CL}}$ on the centerline is considered and shown in Fig. 5. In the LES, the case $h=5 \mathrm{~cm}$ produces an APG strong enough to cause mean flow reversal in the wake, while the case $h=7 \mathrm{~cm}$ is close to flow reversal, but $U_{\mathrm{CL}}$ remains positive.

In the very near wake region, i.e., in the region $x \approx 0.04 \mathrm{~m}$ downstream of the trailing edge of the flat plate, the local maximum of $U_{\mathrm{CL}}$ obtained from the LES is significantly underpredicted by all RANS models. In this region, the flow is subjected to a local acceleration and is in non-equilibrium, which 
is hardly resolved RANS models. This needs to be analysed in future work. Further downstream, for $x>0.1 \mathrm{~m}$, the streamwise decrease of $U_{\mathrm{CL}}$ for the standard RANS models is smaller than for the LES. This becomes even more clear just upstream of the minimum of $U_{\mathrm{CL}}$ for $x>0.3 \mathrm{~m}$. The modification of the SSG/LRR- $\omega$ model using the $S_{\epsilon 4}$-term causes a significant reduction of $U_{\mathrm{CL}}$, and brings the results closer to the LES data. The GGDH modification for the SSG/LRR- $\omega$ model also causes a reduction of $U_{\mathrm{CL}}$. The RC-QCR-extension causes a significant increase of the centerline velocity for the SA model, and a small decrease for the SST model. For the case $h=5 \mathrm{~cm}$, the magnitude of negative velocity in the region of flow reversal cannot be reached by any of the RANS models. Only the SSG/LRR- $\omega$ model with $S_{\epsilon 4}$-term predicts a small region of reverse flow. For the case $h=7 \mathrm{~cm}$, the minimum centerline velocity predicted by the SSG/LRR- $\omega$ model with $S_{\epsilon 4}$-term is close to the LES data, albeit even a little lower.
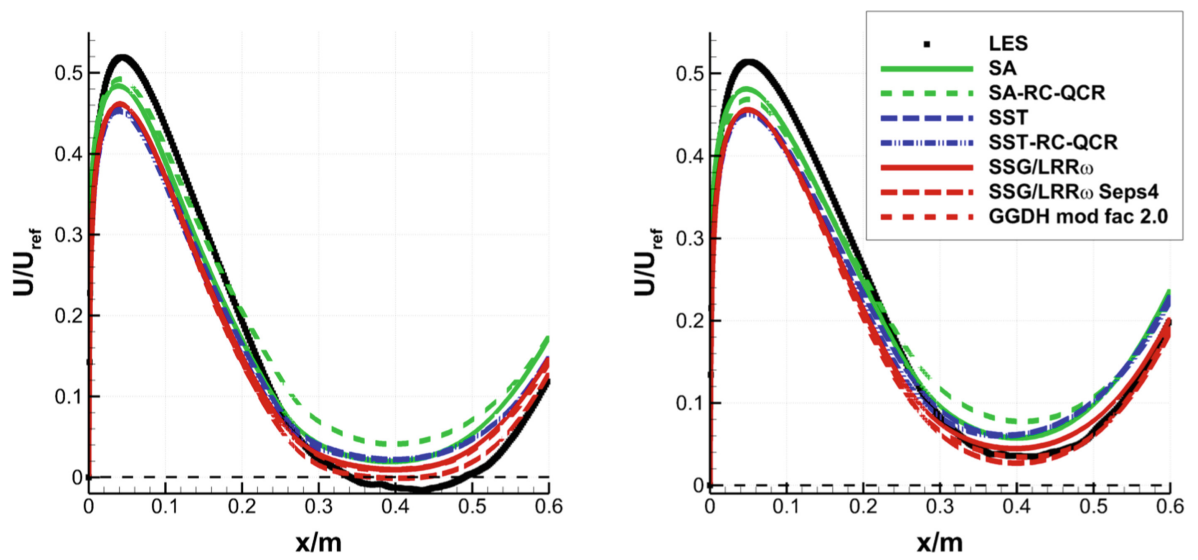

Fig. 5. Centerline velocity for $h=5 \mathrm{~cm}$ (left) and $h=7 \mathrm{~cm}$ (right)

\subsection{Reynolds Stresses}

Figure 6 shows the profiles of the Reynolds stress $\overline{u^{\prime} v^{\prime}}$ in $y$-direction at two different streamwise positions $x=0.2 \mathrm{~m}$ and $x=0.4 \mathrm{~m}$. The eddy-viscosity based RANS models tend to overestimate the magnitude of $\overline{u^{\prime} v^{\prime}}$ more than the SSG/LRR- $\omega$ model. The RC-QCR-extension for SA and SST increases the maximum of $\overline{u^{\prime} v^{\prime}}$ compared to the original model at $x=0.2 \mathrm{~m}$. The modification of the SSG/LRR- $\omega$ using the $S_{\epsilon 4}$-term causes a significant overall reduction of $\overline{u^{\prime} v^{\prime}}$, except near the outer edge of the wake. The GGDH modification causes a similar reduction of $\overline{u^{\prime} v^{\prime}}$ and increases $\overline{u^{\prime} v^{\prime}}$ near the outer edge of the wake. This improves the agreement with the LES data near the outer edge of the wake. 

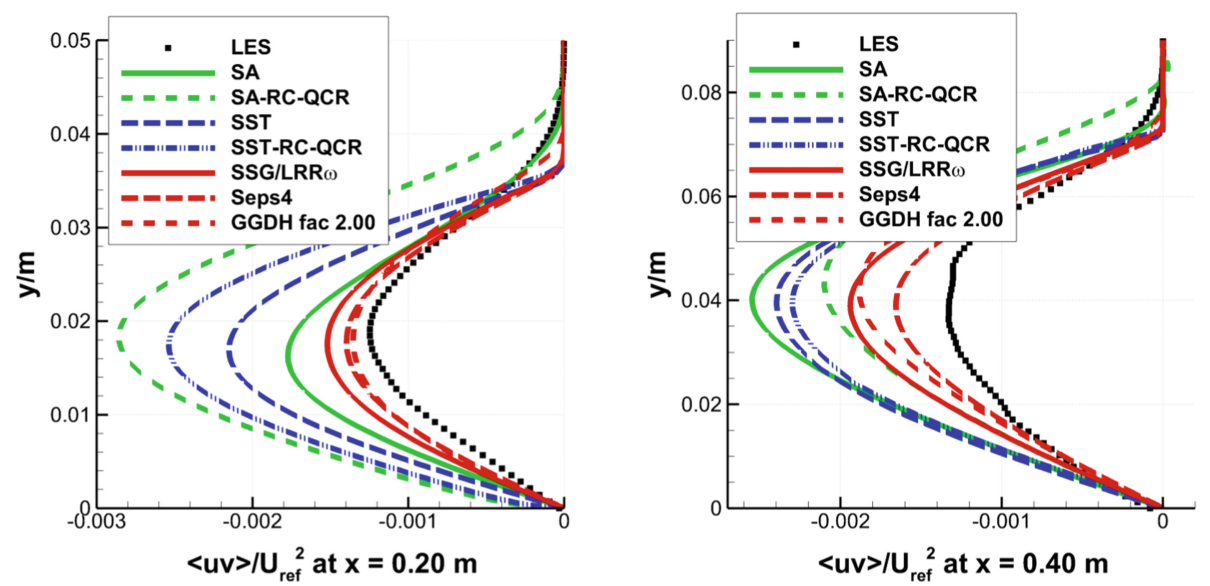

Fig. 6. Profiles of Reynolds stress $\overline{u^{\prime} v^{\prime}}$ for $h=7 \mathrm{~cm}$ at $x=0.2 \mathrm{~m}$ (left) and $x=0.4 \mathrm{~m}$ (right).

\subsection{Ratio of Production to Dissipation of Turbulence}

The LES data reveal that the most relevant terms in the balance equation for the turbulent kinetic energy are production $P_{k}$ and the dissipation $\epsilon_{k}$. The ratio of $P_{k} / \epsilon_{k}$ plays a central role in the streamwise evolution of the Reynolds stresses, and helps to gain further understanding in the effect of the $S_{\epsilon 4}$-term. The results are shown in Fig. 7.

Three regions of the wake (in wall-normal direction) can be distinguished for the comparison between the SSG/LRR- $\omega$ results and the LES data. The first region is the centerline region $(y<0.005 \mathrm{~m})$. The second region is the so-called shear center region of the largest mean velocity gradient in the middle part of the wake $(0.02 \mathrm{~m}<y<0.03 \mathrm{~m})$. The third region is the outer edge of the wake. Here the $y$-values of the different regions are for $x=0.2 \mathrm{~m}$, see Fig. 7 (left).

At $x=0.2 \mathrm{~m}$, the SSG/LRR- $\omega$ model significantly overpredicts $P_{k} / \epsilon_{k}$ both in the centerline region and in the shear center region. For the SSG/LRR- $\omega$ with $S_{\epsilon 4}$-term, $P_{k} / \epsilon_{k}$ is significantly reduced both in the centerline region and in the shear-center region at $x=0.2 \mathrm{~m}$. Further downstream, at $x=0.3 \mathrm{~m}, P_{k} / \epsilon_{k}$ is still a little overpredicted in the shear center region. Hence, overall, the $S_{\epsilon 4}$ modification reduces $P_{k} / \epsilon_{k}$ and brings the prediction closer to the LES data.

An additional indication that it is the shear center region which is of crucial importance can be inferred from $P_{k} / \epsilon_{k}$ for the SST model. The SST model overpredicts $P_{k} / \epsilon_{k}$ in the shear center region, whereas SST-RC-QCR is closer to the LES. This is in agreement with the higher values for $U_{C L}$ for the SST model compared to SST-RC-QCR in Fig. 5 (left) for $0.2 \mathrm{~m}<x<0.3 \mathrm{~m}$. The centerline velocity remains much higher for both models at $x=0.4 \mathrm{~m}$ and is even larger than for the SSG/LRR- $\omega$ model, see Fig. 5 (right). 

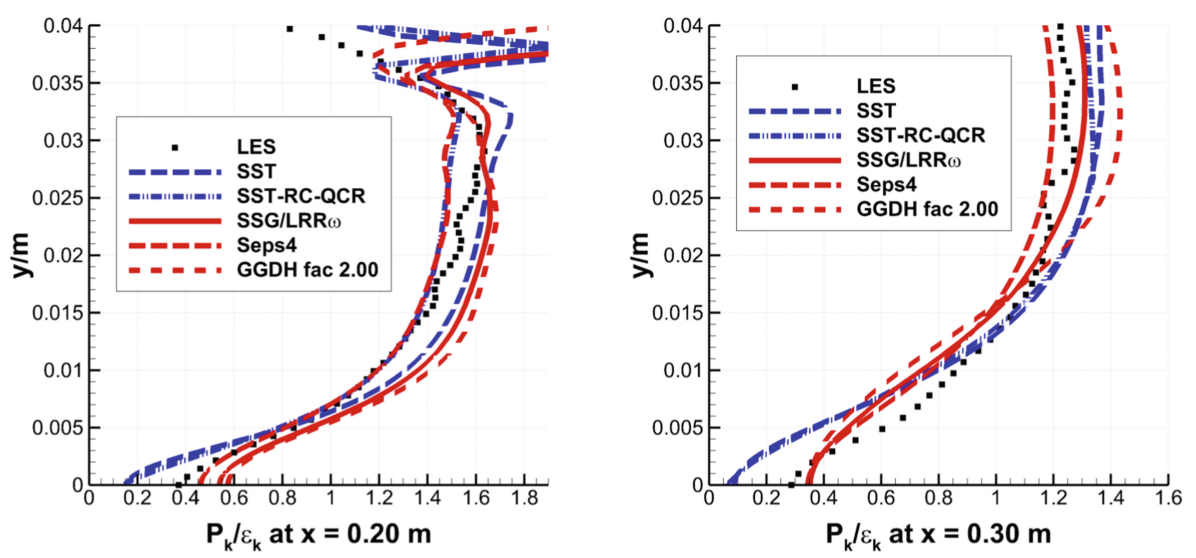

Fig. 7. Profiles of the production to dissipation ratio $P_{k} / \epsilon_{k}$ of turbulent kinetic energy at $x=0.2 \mathrm{~m}$ (left) and $x=0.4 \mathrm{~m}$ (right) for the case $h=7 \mathrm{~cm}$.

\section{Conclusion}

The present work describes the results of RANS computations of a turbulent wake flow in adverse pressure gradient with the use of the SA, SST and SSG/LRR- $\omega$ turbulence models. The models were found to underpredict the onset of flow reversal in the wake. The comparison with the LES data revealed two shortcomings of the SSG/LRR- $\omega$ model. First, it underpredicts the turbulent transport of the Reynolds stresses. Second, it overpredicts the ratio of production to dissipation, and this becomes most important in the so-called shear center region of the largest mean velocity gradient. Therefore two modifications of the SSG/LRR- $\omega$ were studied, i.e., the modification of the coefficient of the gradient diffusion hypothesis for the turbulent transport of the Reynolds stresses and the sensitization of the dissipation rate to irrotational strains. Both modifications reduce the predicted Reynolds stresses in the shear center region and improve the agreement with the LES data. The results indicate that possibly a single modification term cannot eliminate the differences between the SSG/LRR- $\omega$ model and the LES reference data.

In the very near wake region just downstream of the trailing edge, the strong mean flow acceleration observed in the LES is significantly underpredicted by all RANS models. This issue needs to be studied in future research.

Acknowledgement. The funding of the project "Wake Flows in Adverse Pressure Gradient" by DFG and RBRF (Grants No. RA 595/26-1, 17-58-12002 and KN $888 / 3-1)$ is gratefully acknowledged. 


\section{References}

1. Breitenstein, W., et al.: A wind tunnel experiment for symmetric wakes in adverse pressure gradients. In: AIAA Paper 2019-1875 (2019)

2. Burnazzi, M., et al.: Characterization of off-surface separation caused by adverse pressure gradient in a turbulent wake. In: 21th STAB/DGLR Symposium on New Results in Numerical and Experimental Fluid Mechanics, 21, STAB/DGLR Symposium 2018, 6-8 November 2018, Darmstadt, Deutschland (2018)

3. Driver, D.M., Mateer, G.G.: Wake flow in adverse pressure gradient. Int. J. Heat Fluid Flow 23, 564-571 (2002)

4. Eisfeld, B., Rumsey, C., Togiti, V.: Verification and validation of a second-momentclosure model. AAIA J. 54, 1524-1541 (2016)

5. Grenson, P., Probst, A.: Implementation and validation of a near-wall reynoldsstress model based on the homogeneous specific dissipation rate for application to aerodynamic flows. In: Proceedings of the 10th PEGASUS-AIAA Student Conference (2014)

6. Guseva, E., et al.: Experimental/numerical study of turbulent wake in adverse pressure gradient. In: Hoarau, Y., Peng, S.H., Schwamborn, D., Revell, A., Mockett, C. (eds.) Progress in Hybrid RANS-LES Modelling. Notes on Numerical Fluid Mechanics and Multidisciplinary Design, vol. 143, pp. 401-412. Springer, Cham (2020). https://doi.org/10.1007/978-3-030-27607-2_33

7. Hanjalic, K., Launder, B.E.: Sensitizing the dissipation equation to irrotational strains. J. Fluid Eng. T. ASME 102, 34-40 (1980)

8. Probst, A., Radespiel, R.: Implementation and extension of a near-wall ReynoldsStress Model for application to aerodynamic flows on unstructured meshes. In: AIAA 2008-770 (2008)

9. Shur, M., Strelets, M., Travin, A.: High-order implicit multi-block Navier-stokes code: ten-years experience of application to RANS/DES/LES/DNS of turbulent flows (2004). In: 7th Symposium on Overset Composite Grids and Solution Technology, Invited Lecture, Huntington Beach, CA, October (2004). http://cfd.spbstu. ru/agarbaruk/c/document_library/DLFE-42505.pdf

10. Shur, M., Strelets, M., Travin, A.: Improved embedded approaches. Notes Numer. Fluid Mech. Multidiscip. Des. 134, 51-87 (2017)

11. Shur, M.L., Spalart, P.R., Strelets, M.K., Travin, A.: A hybrid RANS-LES approach with delayed-DES and wall-modelled LES capabilities. Int. J. Heat Fluid Flow 29, 1638-1649 (2008)

12. Tummers, M.J., Hanjalic, K., Passchier, D.M., Henkes, R.A.W.M.: Computations of a turbulent wake in a strong adverse pressure gradient. Int. J. Heat Fluid Flow 28, 418-428 (2007)

13. Ying, S.X., Spaid, F.W., McGinley, C.B., Rumsey, C.L.: Investigation of confluent boundary layers in high-lift flows. J. Aircraft 36(3), 550-562 (1996) 